\title{
Adaptively modulated optical fiber link in IM-DD Systems
}

\author{
Pavan Kumar Shukla ${ }^{[1]}$, R.K.Singh ${ }^{[2]}$, S. A. Imam ${ }^{[3]}$ \\ ${ }^{1}$ (Department of Electronics \& Communication Engineering, RKGIT, Ghaziabad, INDIA) \\ ${ }^{2}$ (Uttarakhand Technical University, Deharadun, INDIA) \\ ${ }^{3}$ (Jamia Milia Islamia University, Delhi, INDIA)
}

\begin{abstract}
:
Adaptive modulation is introduced in the field of optical OFDM to motivate the current research carried out in infrared wireless technologies. The proposed all optical OFDM system is described in Section II. Section IV presents the IM/DD systems for the cost effective solution of the optical OFDM system design considerations that should be followed to calculate the system's parameters. Here optical orthogonal frequency division multiplexing systems using direct detection (DD) individually are also described which are suitable for cost-effective applications. A scheme based on discrete Hartley transform (DHT) is proposed and compared to standard Optical OFDM implementation. Both asymmetrically clipped (AC) and DC biased solutions are given in this paper for intensity-modulated DD systems. We show that $\mathrm{AC}$ is a power efficient technique that can be implemented without Hermitian symmetry constraint. For the purpose of getting same performance level the FFT-based Optical OFDM is achieved.
\end{abstract}

Keywords - Asymmetrically clipping, Cyclic Prefix, DFB Lasers, Direct detection, Discrete Hartley transform (DHT), Inter symbol Interference (ISI), Inter channel Interference (ICI), Intensity modulation, Orthogonal Frequency Division Multiplexing .

\section{INTRODUCTION}

In an OFDM system, a serial high data rate data stream is split up into a set of low data rate substreams. The total channel bandwidth is divided into a number of orthogonal frequency sub-channels and each of these low data rate substreams is modulated on a separate sub-channel. The orthogonality is achieved by selecting a special equidistant set of discrete carrier frequencies. It can be shown that, this operation is conveniently performed by the Inverse Fast Fourier Transforms (IFFT). At the receiver, the Fast Fourier Transform (FFT) is used to de-multiplex the parallel data streams . In current research, optical orthogonal frequency division multiplexing is proposed to combat dispersion in optical fiber media [13]. The authors in $[14,15]$ presented the theoretical basis for coherent optical OFDM systems in direct up/down conversion architecture. In [16], the authors have given the basic idea of optical OFDM systems to reduce the interchannel interference which is due to multipath propagation. It is also shown that Optical Orthogonal Frequency Division Multiplexing outperformed RZ-OOK transmission in high-speed optical communication systems in terms of transmission distance and spectral .The system starts with the serial high data rate input which then passes to a serial to parallel $(\mathrm{S} / \mathrm{P})$ block similar to the conventional OFDM system. The all optical OFDM system differs from the conventional OFDM system in the conversion of the low data rate parallel substream into optical signals and performing the IFFT techniques optically rather than electrically. Recent progress of digital signal processing circuit has made it possible to implement the IFFT in wireless communication systems. However, this scheme cannot be applied to the optica communications as the data rate is beyond the digital signal processing speed capabilities.

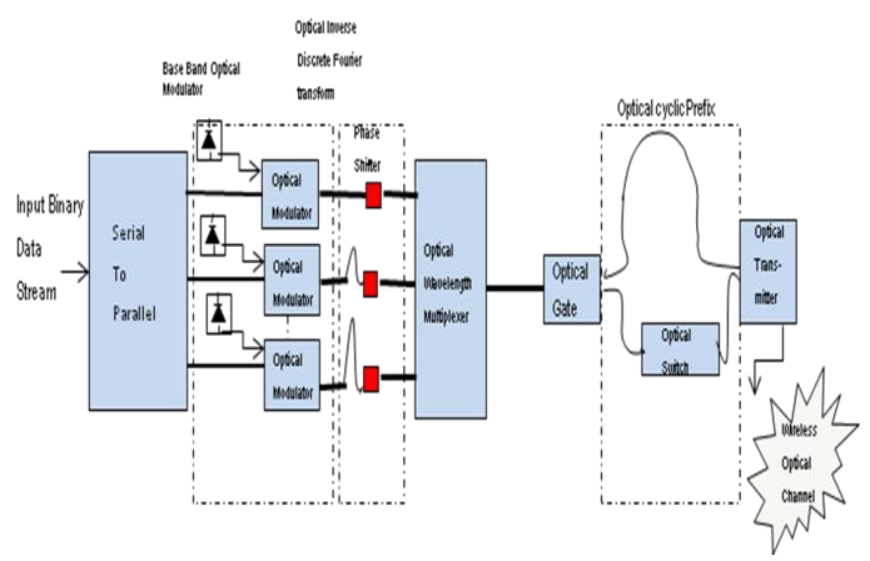

Fig. 1 Optical OFDM transmitter. 


\section{Pavan Kumar Shukla, R.K.Singh, S. A. Imam / IOSR Journal of Engineering (IOSRJEN) www.iosrjen.org \\ ISSN : 2250-3021}

Vol. 2 Issue 2, Feb.2012, pp. 331-337

The low rate parallel sub stream is converted to an optical signal using electrical to optical conversion. This is followed by modulating each optical sub stream using any type of optical modulation techniques as discussed in [13]. All the optical modulators in Figure 2 have the same optical wavelength and are using the same DFB lasers as light sources. The optical conversion and modulation is called baseband optical modulator. The baseband optical modulator is followed by an optical IFFT [14], which consists of fiber delay lines and phase shifters. The number of fiber delay lines is equal to the parallel sub streams which also correspond to number of sub-carriers in the conventional OFDM. The delay lines realize orthogonality by having different lengths. The phase shifters implement the different sub-carriers that are orthogonal to each other and thus will be similar to IFFT done by DSP kits. In conventional OFDM, the output of the IFFT is added together. This is implemented optically using the optical coupler. A cyclic prefix (CP) should be added to overcome the ISI and inter-carrier interference (ICI) [14]. The CP is a crucial feature of OFDM introduced to overcome the multi-path channel effects through which the signal is propagated. The basic idea is to replicate a part of the OFDM time-domain waveform from back to front to create a guard period. The duration of the guard period should be greater than the worst case delay spread of the multi-path environment [15]. This is a challenging technique in optical signals as it is difficult to optically copy and paste.

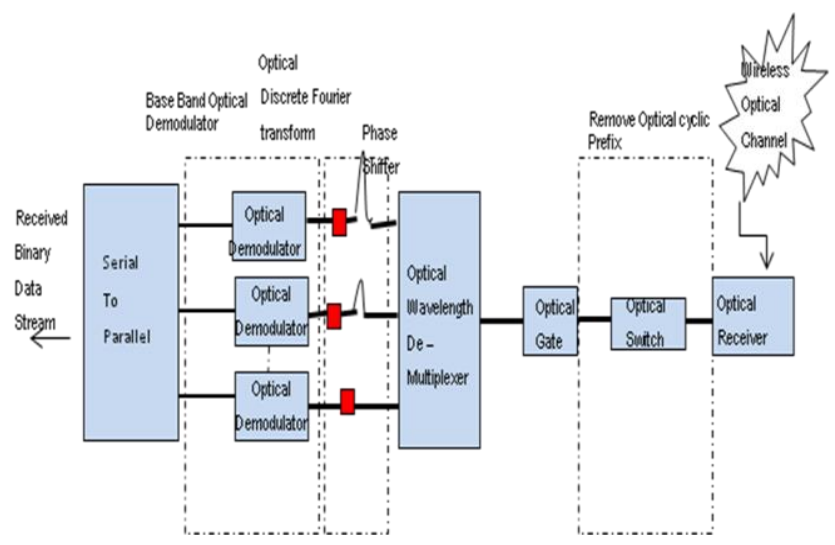

Fig. 2 Optical OFDM Receiver .
This can be overcome using optical gates and what we called optical cyclic prefix. The optical cyclic prefix is divided into two branches using an optical coupler; the first branch is a fiber delay line and the second branch is an optical switch. The optical switch is used to copy the last part of the active ray period and paste it to the front of the optical ray by an optical coupler after it is delayedby a symbol period. The delay is done using the first branch after the coupler. Optical transmitter is used to modulate the OOFDM signal to be suitable for transmission in wireless optical channel[16]. At the receiver side, optical OFDM signal is detected by an optical receiver and then the optical cyclic prefix is removed. The IFFT and optical demodulator are performed to get the corresponding transmitted bit streams the value of non-directed indoor infrared channels ranges from 5 to $20 \mathrm{~ns}$. The symbol duration Ts, must be set much larger than the guard time. A practical design choice for the symbol time is to be at least five the guard time.

\section{OVERSAMPLING}

One of the main advantage of OFDM is that oversampling can be realized by unmodulated subcarriers. This principle can be seen from the electrical output spectrum of an arbitrary waveform generator as shown in Fig. 4. The sampling rate of the AWG is $10 \mathrm{GHz}$ and as such the Nyquist frequency is $5 \mathrm{GHz}$. Oversampling in this example is realized by only modulating 190 of the 256 subcarriers. The 66 subcarriers at high frequencies are left unmodulated, realizing an oversampling factor of 1.34. Because of the oversampling a spectral gap of $\sim 2.5 \mathrm{GHz}$ is present in between the OFDM signal and the aliasing products. An electrical low pass filter can subsequently be used to remove the aliasing products.

Electrical spectrum of an OFDM signal that is generated by an AWG. Compared to single-carrier transmission with coherent detection, optical OFDM reduces the requirements on the sampling rates of analog-to-digital converters (ADC). Whereas in a single carrier receiver often 2-times over-sampling is used for the ADC [10], the minimal practical oversampling resolution is 1.5 -times. With PDM-QPSK 


\section{Pavan Kumar Shukla, R.K.Singh, S. A. Imam / IOSR Journal of Engineering (IOSRJEN) www.iosrjen.org \\ ISSN : 2250-3021}

Vol. 2 Issue 2, Feb.2012, pp. 331-337

this results in an $\mathrm{ADC}$ sampling rate in the range of 42 GSPS for 100GbE. In OFDM redundancy is introduced with the cyclic prefix and training symbols. This overhead can not only be used to overcome inter-symbol interference but also simplifies synchronization and enables the ADC sampling rate to be reduced to about 1.3 times the baud-rate, i.e., about 35 GSPS for PDM-OFDM [10].

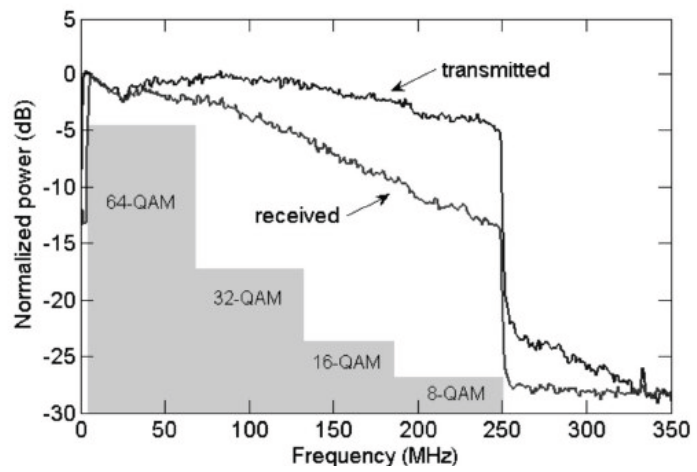

Fig. 3: Transmitted and received (after $50 \mathrm{~m}$ SI-POF) electrical DMT spectra together with applied adaptive QAM

\section{THE FUTURE: INDUSTRIAL ASPECTS OF OPTICAL OFDM}

Optical OFDM for fiber-optic applications has not yet been commercialized. In this section three applications will be discussed for potential commercialization of Optical OFDM in metro and/or long-haul applications. 100 Gigabit Ethernet (100 $\mathrm{GbE}$ ) is considered to become the next generation Ethernet standard for high-capacity backbone networks. It has recently been shown that PDMOFDM is a suitable modulation format for this application[14,15].

However, the dominating modulation format for $100 \mathrm{GbE}$ is single-carrier PDM-QPSK. For PDMOFDM to break through it must offer significant advantages with respect to PDM-QPSK. PDMOFDM offers similar performance to PDM-QPSK, but requires in addition the development of DACs at the transmitter. It has as well been shown that PDMOFDM is not very well suited for deployment on legacy systems as XPM generated by OFDM disturbs its neighboring channels. As such we conjecture that for 100GbE backbone PDM QPSK will be the dominating modulation format.

\section{ADAPTIVELY MODULATED OFDM}

A number of numerical and experimental studies of optical OFDM transmission over MMF have recently been reported in the literature, e.g. [5, 10-14]. In one of the most comprehensive studies, Jin et al. carried out a statistical investigation of the transmission performance of optical OFDM in MMF-based links by numerical simulation [12]. 1000 worst-case MMF links with $3 \mathrm{~dB}$ bandwidths varying between 220 and $490 \mathrm{MHz} \cdot \mathrm{km}$ were considered. OFDM signals with 31 sub-channels were generated with a directly modulated laser. In this study, adaptive modulation was used, where, for each sub-carrier, the modulation format was chosen according to its SNR, from DBPSK, DQPSK, 16 to 256-QAM or no data. All sub-carriers carrying data had the same (average) power. Numerical simulations were performed in a statistical study with a large number of worst-case MMF transfer functions, showing that with optical OFDM, a capacity of more than $30 \mathrm{Gbit} / \mathrm{s}$ over 300 meters could be achieved for over $99.5 \%$ of MMF, based on links installed today. $100 \mathrm{Gbit} / \mathrm{s}$ capacity could be supported in $99.5 \%$ of links of 150 meters length Lee et al. experimentally demonstrated OFDM transmission over $500 \mathrm{~m}$ and $1 \mathrm{~km}$ of MMF [13]. The system used a directly modulated VCSEL operating at $850 \mathrm{~nm}$. Using higher order modulation formats, the link capacity was $30 \mathrm{Gbit} / \mathrm{s}$ over $500 \mathrm{~m}$, with 256 sub-carriers and $20 \mathrm{GS} / \mathrm{s}$ signal converter sampling rate. As described above, the QAM order was selected based on the SNR on each sub-carrier, varying from 2 to 7 bits per symbol. The BER averaged across all channels was $2.5 \times 10-4$, which, through the use of a standard Reed-Solomon FEC code, would be reduced to below 10-10. We carried out numerical simulations assessing interconnect capacity using adaptively modulated OFDM, based on the model of signal propagation through MMF described in [14] and [15]. The considered system consisted of a 50/125 $\mu \mathrm{m}$ graded-index fiber with a refractive index profile approximated by a single $\square$ factor varying between 2.02 and 2.06. A VCSEL-like light source was assumed with a spectral line-width of $10 \mathrm{MHz}$ and an $850 \mathrm{~nm}$ wavelength with overfilled 


\section{Pavan Kumar Shukla, R.K.Singh, S. A. Imam / IOSR Journal of Engineering (IOSRJEN) www.iosrjen.org

Vol. 2 Issue 2, Feb.2012, pp. 331-337

launching condition. The chromatic dispersion at this wavelength was $-94 \mathrm{ps} / \mathrm{nm} \cdot \mathrm{km}$ and the fiber attenuation was $2.3 \mathrm{~dB} / \mathrm{km}$. Transmission of a singleband adaptively modulated OFDM signal over this MMF was simulated, assuming $28 \mathrm{GS} / \mathrm{s}$ DSP, 128 point (I)FFT cores which, with 1.28 oversampling, provided up to 50 discrete multitone (DMT) subcarriers. The power at the receiver was assumed to be $1 \mathrm{dBm}$. Figure 2 (left) shows the fiber frequency response after $300 \mathrm{~m}(\square=2.06)$ where modal coupling and differential mode attenuation were neglected. The graph also shows the modulation format used on each band or group of subcarriers to guarantee a BER of less than 10-3.

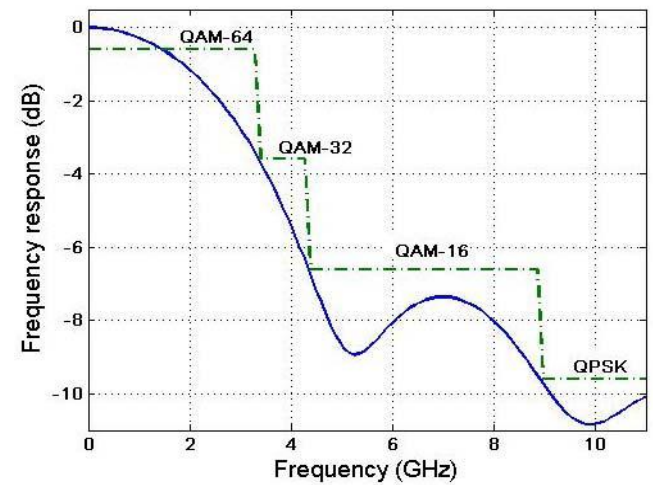

Fig. 4(a): Calculated transfer function of $300 \mathrm{~m}$ of $50 / 125 \mu \mathrm{m}$ graded index MMF at $\square=850 \mathrm{~nm}$, with refractive index profile parameter $\square=2.06$.

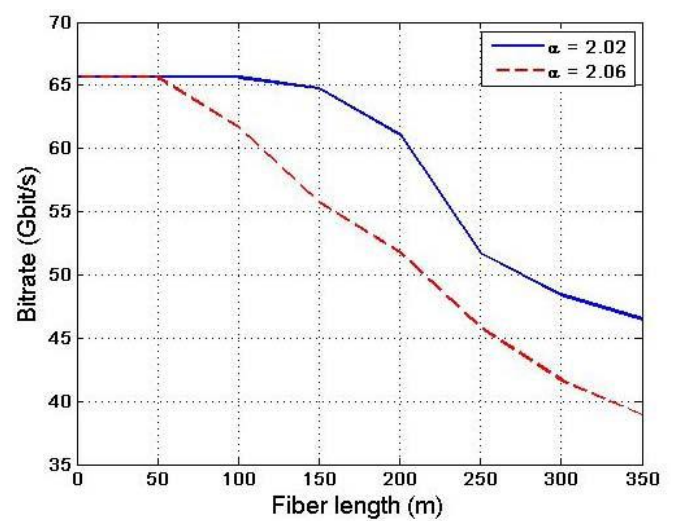

Fig 4(b) Down: Calculated interconnect capacity versus length for two values of refractive index profile parameter $\square$.
The overall bit-rate achieved over $300 \mathrm{~m}$ was 41.7 Gbit/s. This bitrate may be increased by improving the refractive index profile ( $\square$-factor). Figure 2 (right) shows the maximum achievable bit-rate as a function of fiber length for $\square=2.02$ and 2.06. The frequency response of the fiber is flat over a longer distance when $\square=2.02$, and the bit-rate increases to $48.5 \mathrm{Gbit} / \mathrm{s}$ at $300 \mathrm{~m}$. Fig 4(b) Down: Calculated interconnect capacity versus length for two values of refractive index profile parameter $\square$. The subchannel modulation formats used in the OFDM signal used in the transmission simulations are also shown in this plot. As described in the previous section, one of the strengths of OFDM is that it is easy to scale to higher level modulation formats. An interesting application space for optical OFDM is to realize $100 \mathrm{GbE}$ transponders for Metro applications. Cost is here the dominating factor and by using a 16-QAM constellation instead of QPSK (4-QAM), 100GbE PDM -OFDM can be realized with $10 \mathrm{G}$ electronics. A higher constellation size reduces the reach of the transponder, but the reach should be sufficient for both metro and regional applications (up to $\sim 800 \mathrm{~km}$ ). This would significantly educe transponder costs while still offering a large tolerance with respect to chromatic dispersion and PMD [16].

Finally, one can take advantage of the fact that with OFDM it is possible to dynamically set the constellation size of the payload. Taking advantage of this feature one can realize a transponder that can operate at either $100 \mathrm{GbE}$ or $400 \mathrm{GbE}$ by changing the constellation size from 2QAM to 16QAM. Using a PDM-OFDM modulation format, the required bandwidth would be about $60 \mathrm{GHz}$ and as such it would not be suitable for a $50 \mathrm{GHz}$ channel spacing. However, it would provide reach dependent throughput scaling from the ultimate reach with BPSK (2QAM) modulation to the highest data rate with 16 QAM. The main challenge might be that at the client side an interface must be defined that can support both a $100 \mathrm{~Gb} / \mathrm{s}$ and $400 \mathrm{~Gb} / \mathrm{s}$ throughput.

\section{IM/DD SYSTEMS:AC AND DC- BIASED OPTICAL OFDM}

In IM systems, the OFDM signal $s(t)$ is represented by the optical intensity and not by the optical field: the optical power is proportional to $E\{s(t)\}$. 


\section{Pavan Kumar Shukla, R.K.Singh, S. A. Imam / IOSR Journal of Engineering (IOSRJEN) www.iosrjen.org \\ ISSN : 2250-3021}

Vol. 2 Issue 2, Feb.2012, pp. 331-337

Therefore, $s(t)$ must be unipolar. A positive signal can be obtained by adding a DC bias to the real OFDM signal. Usually the bias value $B D C$ is at least two times the signal standard deviation, resulting in an inefficient solution in terms of optical power. A power efficient technique to transmit unipolar signals is the asymmetrically clipping (AC) [17]. Intensity modulation generates a double side-band spectrum, whereas DD systems are more robust against dispersion impairments when combined with SSB modulation. When AC is adopted, only the odd subcarriers are modulated and the OFDM signal can be clipped at zero level without losing information. All the clipping noise falls into the even subcarriers; the symbol sequence can be recovered from the odd subcarriers and the constellation points have the half of the original values. This is still valid for OFDM signal modulated by DHT [14]. The inset shows the received constellation in a back-to-back system using BPSK. Clipping is also required when DC-biased solution is adopted. In fact, if the OFDM signal has high negative picks, although a bias is added, residual negative peaks can be present in the biased signal $h B(t)=h(t)+B D C$, that have to be clipped at zero level for IM. Therefore, an additional noise component affects the signal and, depending on the clipping level, it can severely degrade the transmission[18].

AC and DC-biased techniques trade power and bandwidth efficiency. Indeed, when AC is applied, the electrical signal (proportional to the optical power) results considerably reduced compared to the DC-biased case, even if a minimum bias value is considered, as shown in Fig. 3. However, AC technique requires the double of carriers to transmit the same data per parallel processing of the DCbiased implementation. For both the techniques, the DHT subcarriers supporting data are the double of a real-valued FFT.This paper describes the utility of optical devices and optical sensors in the realization of an all optical OFDM System. The analysis of the OFDM Signal and Optical OFDM signal has a great importance in the utilization of these system in optical wireless networks. The proposed power estimated optical OFDM signal could yield the promising results to overcome the multipath effects and Inter symbol Interference for such type of channels[19] .Its applications have been extended from high freq. radio communication to telephone networks, digital audio broadcasting and digital television terrestrial broadcasting. As portable computers and communication terminals become more powerful and more widely deployed, the demand for high speed wireless communication is increasing.The infrared represents an attractive choice for many short range applications. Its advantages are in the terms of the availability of a wide bandwidth that is unregulated worldwide and that can be reused in a very dense manner, immunity to eavesdropping, ability to achieving very high bit rates, low signal processing complexity, potentially very low cost. Optical OFDM has long been studied and implemented to combat the transmission channel impairments[20]. Its applications have been extended from high freq. radio communications to telephone networks, digital audio broadcasting and digital television terrestrial broadcasting.

\section{Conclusion}

In this paper we have introduced the generation of real-valued OFDM signals which are based on Fourier and Hartley transforms. Basically they are described for cost effective implementation of Optical OFDM using Direct Detection. In this approach Hermitian symmetry constraint is not required as DHT is implemented. This results in a simpler system supporting the double of input symbols. In the case of IM/DD systems AC and DCbiased techniques have been presented which are based on DHT modulation. It is concluded that in FFT based systems, AC is more power efficient and it allows the transmission of unipolar signals without clipping noise. In the same manner the DHT-based scheme uses real lower size constellation to transmit at the same data rate. So it is an alternative Optical OFDM technique for cost-sensitive applications.

\section{ACKNOWLEDGEMENTS}

We find it most pleasant to remember and record our obligations to The Advisor, of RKGIT Ghaziabad, Prof. B.K Gupta \& The Director Dr Laxman Prasad and Prof. K.K.Tripathi, Head,ECE Deptt. for all the supports extended by him to us from time to time. 


\section{Pavan Kumar Shukla, R.K.Singh, S. A. Imam / IOSR Journal of Engineering (IOSRJEN) www.iosrjen.org

Vol. 2 Issue 2, Feb.2012, pp. 331-337

\section{REFERENCES}

[1] A.J. Lowery, L. Du, J. Armstrong: "Orthogonal Frequency Division Multiplexing for Adaptive Dispersion Compensation in Long Haul WDM Systems", Proc. of OFC 2006, pdp39, Anaheim, USA.

[2] W. Shieh, and C. Athaudage: "Coherent Optical Orthogonal Frequency Division Multiplexing,"IEEE, Electron. Letters, Vol. 42, May 2006.

[3] M. Mayrock, H. Haunstein: „Impact of Implementation Impairments on the Performance of an Optical OFDM Transmission System", Proc. Of European Conference on Optical Communications (ECOC), Cannes, France, September 2006.

[4] M. Seimetz, C.-M. Weinert: "Options, Feasibility, and Availability of $2 \times 490^{\circ}$ Hybrids for Coherent Optical Systems", J. of Lightwave Technology, Vol. 24, pp. 1317-1322, 2006.

[5] T. Pollet, M. van Bladel, M. Moeneclaey: "BER Sensitivity of OFDM Systems to Carrier Frequency Offset and Wiener Phase Noise", Trans. on Communications, Vol. 43, pp. 191193, 1995.

[6] W. Shieh, W. Chen, R.S. Tucker: "Polarisation mode dispersion mitigation in coherent optical orthogonal frequency division multiplexed systems", IEE, Electron. Letters, Vol. 42, Aug. 2006.

[7] I. B. Djordjevic and B. Vasic, "Orthogonal frequency division multiplexing for high-speed optical transmission," Opt. Express, vol. 14, no 9, pp. 3767-3775, May 2006.

[8] W. Shieh and C. Athaudage, "Coherent optical orthogonal frequency division multiplexing," Electron. Lett., vol. 42, no. 10, pp. 587-589, May 2006.

[9] K.J. Barker et al:: Entering the Petaflop era: The architecture and performance of roadrunner, in Proc. ACM/IEEE International Conference for High Performance Computing, Networking, Storage and Analysis (SC 2008), Austin, Texas, USA, Nov. 2008.

[10] A. Rylyakov et al.: A 24-channel $300 \mathrm{~Gb} / \mathrm{s} 8.2$ $\mathrm{pJ} / \mathrm{bit}$ full-duplex fiber-coupled optical transceiver module based on a single "holey" CMOS IC, in Proc. OFC 2010, San Diego, California, USA, Mar. 2010, paper PDPA8.

[11] P. Pepeljugoski et al.: Low power and high density optical interconnects for future supercomputers, in Proc. OFC 2010, San Diego, CA, USA, Mar. 2010, paper OthX2.
[12] J. M. Tang et al.: Transmission performance of adaptively modulated optical OFDM signals in multimode fiber links, IEEE Photon. Technol. Lett. 18, 205 (2006).

[13] Y. Benlachtar et al.: Generation of optical OFDM signals using $21.4 \mathrm{GS} / \mathrm{s}$ real time digital signal processing, Optics Express 17, 17658 (2009).

[14] F. Buchali et al.: Realisation of a real-time $12.1 \mathrm{~Gb} / \mathrm{s}$ optical OFDM transmitter and its application in a $109 \mathrm{~Gb} / \mathrm{s}$ transmission system with coherent reception, in Proc. ECOC'09, Vienna, Austria, Sept 2009, PD2.1.

[15] N. Kaneda et al:: Real-time 2.5 GS/s coherent optical receiver for $53.3-\mathrm{Gb} / \mathrm{s}$ sub-banded OFDM, J. Lightwave Technol. 28, 494 (2010).

[16] R. P. Giddings et al.: Experimental demonstration of a record high $11.25 \mathrm{~Gb} / \mathrm{s}$ real-time optical OFDM transceiver supporting $25 \mathrm{~km}$ SMF end-to-end transmission in simple IMDD systems,

[17] N. E. Jolley et al.: Generation and propagation of a $1550 \mathrm{~nm} 10 \mathrm{~Gb} / \mathrm{s}$ optical orthogonal frequency division multiplexed signal over $1000 \mathrm{~m}$ of multimode fiber using a directly modulated DFB, in Proc. OFC 2005, San Diego, California, USA, Mar. 2005, paper OFP3.

[18] X. Q. Jin et al.: Statistical investigation of the transmission performance of adaptively modulated optical OFDM signals in multimode fiber links, J. Lightwave Technol. 26, 3216 (2008).

[19] S. C. J. Lee et al.: Discrete multitone modulation for high-speed data transmission over multimode fibers using 850-nm VCSEL, in Proc. OFC 2009, San Diego, California, USA, March 2009, paper OWM2. 